Referencia para citar este artículo: Londoño-Vásquez, D. A., \& Bermúdez, H. L. (2018). Niveles de literacidad en jóvenes universitarios: entrevistas cualitativas y análisis sociolingüístico. Revista Latinoamericana de Ciencias Sociales, Niñez y Juventud, 16(1), 315-330. doi:10.11600/1692715x.16119

\title{
Niveles de literacidad en jóvenes universitarios: entrevistas cualitativas y análisis sociolingüístico*
}

\author{
DaVid Alberto Londoño-VÁSQUeZ ${ }^{*}$ \\ Profesor Institución Universitaria de Envigado, Colombia. \\ HÉCTOR L. BERMÚDEZ ${ }^{* * *}$ \\ Chargé de cours Département de Management, HEC Montréal, Canada.
}

\section{Artículo recibido en agosto 29 de 2016; artículo aceptado en octubre 18 de 2016 (Eds.)}

- Resumen (descriptivo): se presentan algunos hallazgos emanados de la investigación denominada "Análisis sociolingüístico de los niveles de literacidad en jóvenes de la Institución Universitaria de Envigado", realizada por uno de los autores, para optar al titulo de "Doctor en Ciencias Sociales, Niñez y Juventud" de la Universidad de Manizales-Cinde. Pretende ser una contribución en una doble dirección. Por una parte, denunciar que es común que los jóvenes comiencen sus estudios universitarios con unos niveles de literacidad extremadamente bajos, lo que exige unos esfuerzos especializados en el proceso de enseñanza/aprendizaje de esta población. Por otra, aportar a los aspectos metodológicos, al examinar las posibilidades que ofrece el análisis sociolingüístico de las entrevistas en la investigación empírica. El diseño de la investigación incluyó una intervención académica realizada a una muestra experimental compuesta por 21 estudiantes que ingresaban a la universidad a los cuales se les pidió que diligenciaran una encuesta elemental de perfil sociocultural. Esto se complementó con 5 entrevistas cualitativas. Se concluye que el contexto biográfico desde la infancia y las instituciones involucradas como la familia y la escuela son preponderantes en la adquisición de las competencias en comprensión lectora, producción escritural y apropiación discursiva.

Palabras clave: joven, estudiante universitario, enseñanza superior, sociolingüística (Tesauro de Ciencias Sociales de la Unesco).

\section{Literacy levels in University Students: Qualitative Interviews and Sociolinguistic Analysis}

- Abstract (descriptive): findings from a research study on sociolinguistic analysis of the literacy levels of students from the University of Envigado are presented in this article, which is based on one of the author's thesis for a Doctorate in Social Science, Childhood and Youth at University of Manizales-Cinde. This paper aims to make two distinct contributions to this field. On one hand, it

Este artículo de investigación científica y tecnológica presenta resultados de la tesis doctoral titulada "Análisis sociolingüístico de los niveles de literacidad en jóvenes de la Institución Universitaria de Envigado", presentada por uno de los autores para optar al título de Doctor en Ciencias Sociales, Niñez y Juventud. Universidad de Manizales-Cinde, aprobada por el Acta de Grado No. 62 del 24 de mayo de 2013. La investigación comenzó en julio de 2011 y terminó en diciembre de 2012, para un total de 18 meses de trabajo de campo y análisis. Área de conocimiento: sociología; subárea de conocimiento: Temas especiales.

** Doctor en Ciencias Sociales, Niñez y Juventud de la Universidad de Manizales-Cinde, docente tiempo completo de la Institución Universitaria de Envigado. Researcher ID: F-8907-2013. Orcid: 0000-0003-1110-7930. Índice H5: 8. Correo electrónico: dalondono@correo.iue.edu.co

*** Chargé de cours Sociología de la empresa HEC-Montréal. Sociólogo de la Universidad Autónoma Latinoamericana, Magíster en Ciencias de la Administración de la Universidad Eafit. Grupo de investigación Comportamiento Organizacional (Comphor), Universidad de Antioquia (Col.). Miembro de la Association canadienne des sociologues et anthropologues de langue française (Acsalf). Orcid: 0000-0003-4369-6135. Índice H5: 6. Correo electrónico: hector-leonel.bermudez@hec.ca 
highlights that it is common that young students start their university studies with extremely low literacy levels, which requires specialized efforts in the learning/teaching process of this population. On the other hand, the study provides some interesting methodological aspects, examining the possibilities offered by a sociolinguistic analysis of interviews in empirical research. The research design included an academic intervention, which was carried out with an experimental sample consisting of by 21 students who were in their first semester of an undergraduate degree. These students filled out a basic sociocultural profile survey, which was complemented with 5 qualitative interviews. In conclusion, the study identifies that a student's biographical context since infancy and the institutions involved in their lives, such as family and school, are prevailing factors in the acquisition of reading comprehension and writing production competencies and discursive appropriation.

Key words: youth, university students, superior teaching, sociolinguistics (Social Sciences UnescoThesaurus).

\section{Níveis de literacidade em jovens universitários: entrevistas qualitativas e análise sociolinguística}

- Resumo (descritivo): São apresentados alguns achados que fazem parte da pesquisa chamada de "Análise sociolinguística dos níveis de literacidade em jovens da Institución Universitaria de Envigado", realizada por um dos autores, para a obtenção do título de "Doutorado em ciências sociais, infância e juventude" da Universidade de Manizales-Cinde. O trabalho visa ser uma contribuição em dupla direção. No primeiro lugar, visa expor o fato de ser comum para os jovens o começo do ensino universitário com níveis de literacidade extremamente baixos, o que exige esforços especiais no processo de ensino/aprendizado para essa população. Alem disso, a pesquisa aporta aspectos metodológicos, ao examinar as possibilidades oferecidas pela análise sociolinguística nas entrevistas da pesquisa empírica. O projeto de investigação incluiu uma intervenção acadêmica feita com uma amostra experimental de 21 estudantes que entraram na universidade para a qual foram convidados, que deve processar uma pesquisa elementar de perfil sociocultural. Esta foi complementada por cinco entrevistas qualitativas. Concluiu-se que o contexto biográfico desde a infância e instituições envolvidas neste contexto como a família e a escola são preponderantes na aquisição de competências em compreensão de leitura, produção literária e apropriação discursiva.

Palavras-chave: jovens, estudantes universitários, ensino superior, sociolinguística (Thesaurus de Ciências Sociais da Unesco).

\section{-1. Introducción. -2. Marco teórico. -3. Metodología. -4. Resultados. -5. Conclusiones. -Lista de referencias.}

\section{Introducción}

Las investigaciones sobre problemas relacionados con los procesos de enseñanza/ aprendizaje en la población joven son innumerables y extremadamente variadas. Caben aquí, estudios tan disímiles como aquellos sobre la interferencia lingüística (Buitrago, Ramírez \& Ríos, 2011) o la discriminación lingüística (Hernández-Rosete \& Maya, 2016); sobre la metacognición en la comprensión lectora (Jaramillo, Montaña, \& Rojas, 2006) o la argumentación metacognitiva (Sánchez-
Castaño, Castaño-Mejía, \& Tamayo-Alzate, 2015). Esto, por solo mencionar algunos pocos. Es necesario pues delimitar. Así, este artículo se concentrará, exclusivamente, en el campo de la literacidad. Esta es entendida aquí en el sentido de Cassany (2006, p. 38), cuando advierte que esta "abarca todo lo relacionado con el uso del alfabeto: desde la correspondencia entre sonido y letra hasta las capacidades de razonamiento asociadas a la escritura". Es decir, la literacidad como mucho más que la simple capacidad para leer y escribir uniendo estos procesos. Esta incluye además, una serie de habilidades 
que se desarrollan en culturas y contextos muy específicos, acorde con los cuales actúan las personas. En este artículo entonces se presentan algunos hallazgos obtenidos en una de las etapas de la tesis doctoral titulada Análisis sociolingüistico de los niveles de literacidad en jóvenes de la Institución Universitaria de Envigado (IUE) (Londoño-Vásquez, 2013). Concretamente, se trata de la fase en la que se llevó a cabo un conjunto de entrevistas cualitativas. El objetivo de la tesis fue describir los niveles de literacidad y la posible correlación con los factores sociales como el estrato social, la edad y el sexo, el nivel educativo de los padres, y la influencia de la escuela en los estudiantes participantes. Esta inquietud no es nueva en absoluto. La literacidad en jóvenes universitarios ha sido examinada, entre muchos otros aspectos, con relación a la alfabetización de sus padres (Philliber, Spillman, \& King, 1996), a las estrategias populares de enseñanza del pasado (Stahl, King, \& Eilers, 1996), a literacidad crítica (Behrman, 2006) o a los asuntos identitarios y de relaciones de poder (Zavala, 2009).

Así, para dar cuenta del objetivo de la presente investigación, se acudió a la riqueza teórica y metodológica del análisis sociolingüístico. La acumulación lograda por investigadores de diferentes tradiciones al interior de este campo de estudio (Forlot, \& Martin, 2014; Jablonka, 2016; Lawson \& Sayers, 2016), brinda, en la práctica, oportunidades de interpretación de enorme fecundidad heurística y de total pertinencia para estudiar un problema como el de la literacidad en jóvenes universitarios.

El lector encontrará entonces, además de la presente introducción, cuatro apartados más. Uno sobre ciertos aspectos teóricos de los estudios de la literacidad y la importancia del análisis sociolingüístico como apoyo metodológico para examinarla en los jóvenes que comienzan la universidad. Luego, se presentan los principales aspectos de la metodología llevada a cabo en la investigación. Posteriormente se muestran algunos resultados emanados de la mencionada etapa del trabajo de terreno de la misma. Por último, el artículo, además de anunciar algunas implicaciones

Rev.latinoam.cienc.soc.niñez juv 16 (1): 315-330, 2018 http://revistalatinoamericanaumanizales.cinde.org.co doi: $10.11600 / 1692715 \times .16119$ prácticas, cierra con unas pocas conclusiones que habría que entender mejor como valiosas oportunidades de investigación.

\section{Marco teórico}

La literacidad es una noción incluyente. Abarca el código escrito, los géneros discursivos, los roles de autor y lector, las formas de pensamiento, la identidad y el estatus de individuo o de colectivo, así como los valores y las representaciones culturales. Todos ellos, elementos que rigen la interacción de un sujeto con respecto a las diferentes prácticas discursivas, las cuales no solo son el producto del conocimiento (campo disciplinario) sino que tienen un carácter sociocultural, donde el mismo conocimiento se ve afectado por ese contexto. Tan es así que Kress (2003) propone la literacidad como un concepto tripartito que no puede separarse de los factores sociales, mediáticos y económicos. De hecho, la literacidad ha venido ganando espacios en las discusiones académicas en los últimos 15 años, lo cual ha causado una acumulación en diferentes perspectivas ${ }^{1}$. Igualmente, el radio de problemas de investigación es vasto. Recientemente, algunos autores se han ocupado de explicar, por ejemplo, los fenómenos inherentes a la lectoescritura digital académica (Aguilar, Ramírez, \& López, 2014; Castek \& Beach, 2013; Larson, 2013; Vargas, 2015), a la lectoescritura por imágenes y gráficas (Botzakis, 2016; Rowsell \& Kendrick, 2013), o a la responsabilidad ética de los educadores (Parker, 2013).

Así, en el contexto de la presente investigación, la literacidad es entendida como el conjunto de "prácticas relacionadas, no sólo con la búsqueda eficaz de la información, sino también con su uso y manipulación pertinente" (Aguilar et al., 2014, p. 125); además, con la "comunicación asertiva de tal información enfocada a la resolución de problemas" ( $\mathrm{p}$. 126). Ésta —en la realidad de los jóvenes

$1 \quad$ Al respecto, Londoño-Vásquez (2015) revisa ciertas diferencias entre las perspectivas sociocognitivas (Parodi, 2010), socioculturales (Cassany, 2008), antropológicas (Freebody \& Luke, 1990), lingüísticas (Castañeda \& Henao, 2002) y de estudios críticos (Behrman, 2006). 
universitarios_-, "está condicionada por toda una gama de aspectos referentes al contexto que está viviendo el estudiante" (p. 126). Por eso, algunos investigadores se preocupan por un fenómeno común en la universidad: las dificultades que tienen los estudiantes que ingresan a la formación superior para comprender los textos que leen, para producir textos coherentes y cohesivos, para consolidar discursivamente conceptos propios de sus respectivas carreras y apropiarse del conocimiento. Igualmente, para que critiquen los puntos de vista inscritos en las diversas perspectivas en cuestión y puedan argumentar su propio punto de vista. Pero, en general, "se asume que los estudiantes en la educación superior ingresan a estas instituciones listos para responder a las demandas de literacidad que les exige este nivel" (Zavala, 2009, p. 343). Sin embargo, "con la masificación de la educación superior no hay ninguna garantía de que los estudiantes lleguen preparados para lidiar con la literacidad de tipo académico que se requiere de ellos en estas instituciones" ( $p$. 343). Justamente, este tipo de inquietudes es uno de los interrogantes que nuestra investigación se plantea.

En el apartado metodológico se verá que para dar cuenta del objetivo trazado, en este estudio se privilegió un enfoque cualitativo que incluye, entre otros mecanismos, un conjunto de entrevistas. Sin embargo, la experiencia investigativa en las ciencias sociales indica que una de las dificultades más comunes en las investigaciones cualitativas es, precisamente, el análisis de las entrevistas (Boutet, 2016; Holstein, \& Gubrium, 2016; Kvale, 2011). Este requiere no solo de la adopción de un enfoque teórico que permita construir una metodología adecuada y coherente para lo que se pretende, sino también pasar a la praxis; es decir, materializar el análisis como tal.

Así, la riqueza teórica y metodológica lograda históricamente por los análisis sociolingüísticos, pudo aprovecharse en este trabajo, tanto para el diseño metodológico como para el análisis de la información recolectada.

Grosso modo, la sociolingüística es, según la ya clásica definición de Labov (1976), el estudio de la estructura y la evolución del lenguaje en el seno de un contexto social formado, precisamente, por la "comunidad lingüística". Es necesario, sin embargo, delimitar, puesto que el campo en el que han sido aplicados los análisis sociolingüísticos es bastante amplio. Hace ya cuatro décadas, Laurence Bardin (1977) destacaba, por ejemplo, los "análisis de las comunicaciones de masas" (con su ejemplo del horóscopo de las revistas populares), el "análisis de las respuestas a preguntas abiertas" y el "análisis de resultados a los test de asociaciones de palabras", entre otros. Por su parte, Henri Boyer (2001, pp. 18-21) señala los análisis sociolingüísticos aplicados a la "gestión de las lenguas", el "análisis de la variación sociolingüística en el seno de los grupos", el "análisis de los fenómenos lingüísticos en situaciones de migración", el "tratamiento lexicológico-lexicométrico de los discursos sociales (políticos, sindicales, mediáticos)", el "análisis sociolingüístico de las interacciones verbales", etc. La pertinencia de la sociolingüística puede constatarse, no solamente por el gran número de publicaciones actuales en revistas especializadas en análisis sociolingüísticos (cf. https://lengas.revues. org/ ; http://www.sociodialeto.com.br/; https:// journals.equinoxpub.com/index.php/SS/ index;), sino también en algunas compilaciones tipo Handbook, en las cuales pueden apreciarse los diversos tipos de problemas, los desafíos teóricos y metodológicos, las oportunidades futuras de investigación, la aplicación y el impacto (Forlot \& Martin, 2014; Jablonka, 2016; Lawson \& Sayers, 2016).

En esta investigación en particular, se revisaron varias tradiciones: la Lingüística Textual (Beaugrande \& Dressler, 2007; Calsamiglia \& Tusón, 1999; Cook-Gumperz, 1988; Renkema, 1999), el Análisis Crítico del Discurso (Cassany, 2005a, 2005b, 2008; Freebody \& Luke, 1990; Green, 2001; Van Dijk, 1991, 1999) y la Argumentación Pragmadialéctica (Van Eemeren \& Grootendost, 1990, 1992; Van Eeemeren, Grootendost, \& Snoeck, 2006). De igual forma, este marco de referencia completó así el examen que se había emprendido antes sobre los tres 
principales enfoques de lo que se conoce como "los estudios críticos del discurso" (LondoñoVásquez \& Bermúdez, 2013) y contribuyó a proteger, desde el punto de vista teórico, a la investigación en su conjunto. Sin embargo, una de las mayores contribuciones de esta revisión de las tradiciones sociolingüísticas emerge de los aportes teóricos de autores como Basil Bernstein (1989) y sus nociones de código restringido y elaborado, y Michael Halliday (1982) con la importancia que este autor brinda a la relación lenguaje-educación. Todo lo anterior, favoreció la consolidación teórica y metodológica de la intervención académica.

\section{Metodología}

La IUE ${ }^{2}$, es una institución universitaria pública, localizada en el municipio de Envigado, al sur de Medellín (Colombia). Cuenta con cuatro facultades: una de Derecho, Ciencias Políticas y Jurídicas; otra de Ciencias Empresariales; una tercera de Ingenierías; y una cuarta de Ciencias Sociales. Acorde con la normatividad colombiana de la educación superior, la IUE ofrece programas universitarios de 10 semestres de duración (el semestre I, de enero a junio, y el semestre II, de julio a diciembre). Esto, en jornadas diurnas y mixtas $^{3}$. La presente investigación, se llevó a cabo con un grupo de estudiantes de primer semestre del programa de Psicología (adscrito a la Facultad de Ciencias Sociales) y otro del primer semestre del programa de Derecho (adscrito a la Facultad de Derecho, Ciencias Políticas y Jurídicas). Concretamente, el trabajo de terreno se desarrolló en el marco de dos cursos formales que los estudiantes de los mencionados programas deben cursar puesto que hacen parte de su malla curricular: el "Taller de Lectura y Escritura" y el "Taller de Técnicas Comunicativas", respectivamente. Estos cursos tuvieron una duración de 48 horas presenciales.

2 Para la Institución Universitaria de Envigado (IUE), véase: http://www.iue.edu.co/portal/index.php.

3 Las jornadas diurnas son aquellas en las cuales las clases son en los horarios comprendidos entre las 8:00 a.m. y las 6:00 p.m.; las jornadas mixtas, aquellas que incluyen horarios extremos: desde las 6:00 a.m., hasta las 10:00 p.m.
El diseño de la investigación incluyó dos grandes pasos metodológicos: una intervención académica ${ }^{4} \mathrm{y}$, luego, un conjunto de entrevistas. La primera, fue realizada a una muestra experimental compuesta por 21 estudiantes que ingresaban a la universidad, las entrevistas, a cinco de estas personas. La muestra de la intervención académica se subdividió en dos grupos. Uno, el de los estudiantes del programa de Psicología, el otro, el de los estudiantes del programa de Derecho. Posteriormente, los 21 participantes presentaron dos pruebas (una inicial y otra final). Para la medición de las pruebas se utilizó la rúbrica propuesta por Henao, Londoño-Vásquez, Frías y Castañeda (2011), la cual se centra en tres aspectos: el resumen, la lectura crítica y la argumentación pragmadialéctica. Dicha rúbrica cuenta con 25 variables y permite pasar de lo cualitativo a lo cuantitativo y viceversa dependiendo del interés investigativo ${ }^{5}$. Estas pruebas se realizaron con el ánimo de conocer los niveles de literacidad iniciales y finales de dichos participantes en el primer semestre de sus respectivas carreras.

Igualmente, se pidió a los participantes que diligenciaran una encuesta elemental de perfil sociocultural. Esta encuesta permitió conocer no solo las variables sociales básicas sino también sus principales prácticas lectoras y sus experiencias en el proceso de formación en la lectura, tanto de la familia como de otras personas significativas.

En lo concerniente a la selección de los participantes, cabe mencionarse que el programa de Psicología en IUE, para el semestre 2010II, ofreció tres grupos de Taller de Lectura y Escritura I, cada uno a cargo de un docente diferente. Uno de esos cursos fue asignado a un docente, cuya remarcable experiencia en

$4 \quad$ Por "intervención académica" hay que entender aquí los cursos (el "Taller de Lectura y Escritura" y el "Taller de Técnicas Comunicativas"). Estos se concibieron, ofrecieron y evaluaron como prácticas pedagógicas que se diseñan y llevan a cabo en el aula con el objetivo de subsanar, afianzar o corregir las dificultades en elaboración de resúmenes (sociocognitiva), la realización de una lectura crítica (sociocultural) y la argumentación (pragmadialéctica). ( $\mathrm{Al}$ respecto, véase: IsázigaDavid, Gabalán-Coello, \& Vásquez-Riso, 2014).

5 Una descripción más detallada de dicha rúbrica, de los resultados obtenidos y del análisis realizado se encuentra en LondoñoVásquez (2015). 
los procesos de lectoescritura fue fundamental durante la investigación ${ }^{6}$. Para el caso de este grupo, entre los 10 estudiantes que terminaron el curso, se tuvo en cuenta a seis jóvenes menores de 26 años (dos hombres y cuatro mujeres), uno de estos, una estudiante proveniente de Brasil ${ }^{7}$. Con respecto a la carrera de Derecho, en el semestre 2011-II, el programa ofreció dos grupos por jornada. Uno de ellos estuvo a cargo del mismo "docente colaborador". Para este caso, la muestra consistió en 15 jóvenes (tres hombres y 12 mujeres) que cumplían con el rango de edad establecido para la investigación.

Sibien el análisis de los niveles de literacidad y de las variables sociales proporcionaba una información relevante, era necesario conocer qué pensaban los participantes y el docente colaborador al respecto, puesto que hay algunos resultados cuantitativos que pueden responder a otras variables no contempladas en la investigación. Por tanto, después de analizados los datos recolectados con las encuestas y las pruebas, tanto de entrada como de salida, se invitó a los participantes que voluntariamente quisieran ser entrevistados, siempre y cuando, no fueran mayores de 26 años para la fecha de la conformación del grupo experimental. Del programa de Psicología, solo un hombre y una mujer con estas características quisieron participar; curiosamente, la joven voluntaria era la brasileña que ya se mencionó, la cual llevaba dos años en Colombia cuando comenzó a estudiar en la IUE. Dada la situación, se tomó la decisión de entrevistarla, teniendo en cuenta que si bien no permitiría revisar elementos sociales de Antioquia (Colombia), sí facilitaba comparar algunas tendencias con respecto al núcleo familiar, el estrato social y los procesos de literacidad en la escuela. Con respecto a los participantes del programa de Derecho, de igual forma, solo se tuvo a dos voluntarios con las características planteadas: una mujer y un hombre, pero esta vez, ambos nacidos y educados en Antioquia.

$6 \quad$ El rol de este docente fue, además servir de profesor del "Taller de Lectura y Escritura I", contribuir con su testimonio en una de las cinco entrevistas cualitativas. En adelante, en el artículo se le nombrará como "docente colaborador".

7 Todos los participantes firmaron un acta de consentimiento informado, según los cánones éticos en investigación cualitativa.
Las cinco entrevistas (las dos de Psicología, las dos de Derecho y la del docente colaborador) fueron realizadas por el investigador principal en las instalaciones de la Institución Universitaria de Envigado en el lapso de una semana en el mes de marzo de 2012. Estas entrevistas fueron grabadas y transcritas en su totalidad, con el propósito de facilitar su análisis desde una perspectiva cualitativa como lo es la sociolingüística. Cabe mencionar que los errores gramaticales y de dicción cometidos por los participantes, el docente colaborador y el entrevistador se mantuvieron en la transcripción, al igual que las repeticiones innecesarias. Esto, porque editar para mejorar la estética de la presentación de los hallazgos de la investigación puede ser una idea útil para los asuntos de estilo, pero puede atentar contra la evidencia empírica obtenida a partir del análisis sociolingüístico y entorpecer la interpretación.

En el programa de Psicología se contó entonces con una mujer de 20 años proveniente de Santa Catarina (Sur del Brasil), bilingüe (portugués y español) y que, al momento de la investigación, llevaba viviendo dos años en Envigado. Con respecto al hombre, este es un joven de 26 años, técnico en preservación de cadáveres, oriundo de Sopetrán (Antioquia), que vivió en Copacabana (Antioquia) la mayor parte de su niñez y adolescencia donde terminó su secundaria y que vive en Envigado desde hace más de una década.

En Derecho se contó con una mujer de 21 años, proveniente de San Antonio de Prado (Antioquia). Tan pronto terminó la secundaria comenzó a estudiar en la IUE. El otro participante es un joven que tiene 23 años, ha vivido siempre en Envigado, ha iniciado varias carreras en diferentes universidades, pero nunca las ha terminado. Este participante es el único de los cuatro entrevistados que no aprobó el curso.

Conviene, para terminar este apartado, mencionar las competencias del docente colaborador, puesto que, a partir de ellas, y con la colaboración inmediata del investigador principal, pudo brindarse rigor científico a la investigación. El mencionado docente es Licenciado en Español y Literatura de la 
Facultad de Educación de la Universidad Antioquia, Magíster en Sociología de la Educación de la misma Universidad; realizó una especialización en Logopedia (Terapia del Lenguaje) en el Instituto de Ciencias del Hombre de Madrid y adelantó estudios de doctorado en la Universidad de Lérida. Asimismo, ha participado en seminarios, cursos, eventos y congresos relacionados con la literacidad, tanto en Colombia como en otros países. Lleva 40 años orientando este tipo de cursos en las universidades de Antioquia, de Medellín, de San Buenaventura, Luis Amigó y la Institución Universitaria de Envigado; además, trabajó más de 20 años en el Instituto Técnico Industrial Pascual Bravo, sección de bachillerato. Al respecto de su trayectoria, señala lo siguiente:

Mi experiencia en este tipo de cursos es amplia y el compartir con jóvenes de secundaria y, simultáneamente, con los de la Universidad me permitía ver las dos caras del proceso y constatar la necesidad de darle un giro radical a la manera como se enseña la cultura escrita, tanto en la escuela como en la educación superior. A la vez, ir adecuando el curso de acuerdo con los cambios en las culturas juveniles y a los avances teóricos y metodológicos en literacidad, que han sido bastante significativos en los últimos años, como se puede constatar en la bibliografía sobre el tema (Docente colaborador).

Por tanto, su compromiso con la enseñanza de la lectura y escritura comprendiendo los contextos culturales específicos (premisa fundamental de la sociolingüística) pudo evidenciarse, no solamente por su propio saber acumulado, sino también, potenciarse durante el trabajo de campo de la investigación.

\section{Resultados}

Conviene aclararse que, si bien en la investigación se tuvieron en cuenta cinco parámetros de análisis: la familia, la escuela primaria y secundaria, los problemas académicos, la perspectiva del curso y las recomendaciones a la propuesta (LondoñoVásquez, 2013), por cuestiones de espacio, en este artículo fue necesario centrar la atención,

Rev.latinoam.cienc.soc.niñez juv 16 (1): 315-330, 2018 http://revistalatinoamericanaumanizales.cinde.org.co doi: $10.11600 / 1692715 x .16119$ fundamentalmente en las variables familia y escuela. Igualmente, y como se anunció en la introducción, se presentan aquí, únicamente, algunos resultados emanados del análisis de las entrevistas cualitativas ${ }^{8}$.

Los resultados en la prueba final indicaron que ambos grupos mejoraron, pero de manera bastante desigual. El grupo de estudiantes de Psicología obtuvo resultados más significativos que el de los estudiantes de Derecho en cuanto a la mejoría del nivel de literacidad, lo cual animó a uno de los autores a preguntarse si estos resultados solo se debían a las correlaciones encontradas entre edad, sexo y formación de los padres, o si era posible hallar otras correlaciones que no se habían tenido en cuenta, como las que se desprenden, por ejemplo, del proceso de enseñanza/aprendizaje directamente. Es allí, precisamente, donde el análisis sociolingüístico permitió desentrañar elementos que, con métodos menos rigurosos pueden quedar, fácilmente, aislados o solapados por otros datos.

\subsection{Entrevista a tres participantes colombianos}

La influencia de la familia en los procesos de "socialización primaria" (Berger, \& Luckmann, 2003; Friedman, 2016) es uno de los factores relevantes en el desarrollo de competencias en el lenguaje de cualquier niño. Sin ser deterministas, esto significa que un niño que esté expuesto en casa a procesos de lectura, a discusiones con tinte académico, social, económico o político donde la argumentación hace parte de estas, el acompañamiento de los padres o de los hermanos mayores, iría construyendo bases lingüísticas privilegiadas que le permitirían reflexionar para hablar de su propia realidad (Bernstein, 1989; LondoñoVásquez \& Castañeda, 2011)..$^{9} \mathrm{Al}$ respecto, se le preguntó al docente colaborador si cree que haya una relación entre los niveles de literacidad

$8 \quad$ Los resultados generales aparecen en Londoño-Vásquez (2013); para otros resultados, véase: Londoño-Vásquez (2015).

9 Cabe notarse que aunque tanto los determinantes genéticos, como los culturales son importantes, el hecho de estar en condiciones favorables no siempre garantiza el desarrollo del nivel de literacidad en todos los individuos (Cyrulnik, 2001). 
y la situación sociocultural de los estudiantes, a lo cual respondió:

Aunque esto se puede tomar en sentido negativo, si no reconocemos el papel de lo sociocultural en el manejo del lenguaje, no podremos cambiar la situación de desventaja con que ingresan a la educación en general $\mathrm{y}$, en forma especial, a la universitaria los jóvenes de los estratos más bajos de la población. Esto incide en su capacidad verbal, en la posibilidad de comprar los materiales necesarios para la carrera, en la alimentación, en los desplazamientos para las actividades académicas, en el contacto con la cultura $\mathrm{y}$ en el conocimiento de muchos temas $\mathrm{y}$ realidades que para unos son parte de la cotidianidad y, para los otros, desconocidas. A veces preguntan por el significado de palabras tan comunes para la mayoría, que la misma pregunta genera desconcierto. Por ejemplo, ¿qué es una palabra aguda?, ¿por qué después de punto va mayúscula? (Docente colaborador $)^{10}$.

Nótese en el énfasis de la cita el aporte del enfoque sociolingüístico del docente experto: por una parte, tiene en cuenta algunos aspectos del contexto que determinan el aprendizaje $y$, por otra, el examen que hace del discurso, propiamente dicho, de los estudiantes.

Por otro lado, se tiene a tres participantes que reconocen tener bajos niveles de literacidad para desempeñarse satisfactoriamente en los diferentes cursos que componen la malla curricular de sus respectivos programas académicos. Al preguntarles si consideraban que tenían (o no) un buen nivel de lectura y escritura, los entrevistados respondieron:

Sí, lo he estado mejorando pues anteriormente si tenía muchas falencias (Hombre, Psicología).

No, porque leo súper mal, confundo las palabras. O sea, cuándo estoy leyendo y más en público, me, cómo que me pongo nerviosa y digo una cosa que no..., similar

10 Todos los énfasis de las citas de las entrevistas son agregados por los autores. pues en el nombre y no súper mal, me da mucha pereza leer (Mujer, Derecho).

Más o menos (Hombre, Derecho).

Y su autocrítica no está muy apartada de la realidad, al menos con respecto a los resultados de las pruebas de entrada. Los tres entrevistados obtuvieron puntajes inferiores a 1,0 en una escala de 1,0 a 5,0 . No obstante, los resultados de las pruebas de salida, indican que dos de los tres entrevistados obtuvieron un puntaje superior a la media de la unidad de análisis (Mujer, Derecho: de 0,3 a 1,4; Mujer, Psicología: de 1,0 a 4,0; Hombre, Derecho: de 1,0 a 2,1; y Hombre, Psicología: de 0,3 a 3,3). Esto indica que, si bien no es posible para las diferentes instituciones de educación superior contar única y exclusivamente con estudiantes "ideales", uno de los retos fundamentales en la enseñanza de la lectoescritura en los primeros niveles universitarios consiste en mantener el compromiso del estudiante frente a sus metas y contribuir a que ensayen a subsanar las fallas y dificultades bien sean estas de origen social, económico o familiar, donde la lectura y la escritura resultan dos herramientas vitales.

Por esto, a los entrevistados se les preguntó sobre cómo fue la relación en casa con respecto a la lectura y la escritura y sus respuestas fueron las siguientes:

Pues, la exigencia por parte de mi padre fue mucha porque pues él es poeta y ha escrito pues como libros, entonces él siempre era como que pues que había que hacer mucho énfasis en eso. Pero también, pero los fines de semana pues más que todo porque en semana él no estaba pues como presente, entonces mi mamá era la que nos cuidaba y pues no era así como una motivación pues así como... (Hombre, Psicología).

Muy mal, porque mi papá y mi mamá trabajaban. Entonces, a nosotros nos dejaban en una guardería y no...; allá no le enseñan a uno... Pues no tengo una razón pa' acordarme de cosas, pero no, súper mal (risas) (Mujer, Derecho).

Pues, mi papá es buen lector, mi papá lee bastante. Y mi mamá, pues más o menos, y ya es lo que más de pronto como sí y ya aparte en el colegio, los amigos, de 
pronto me recomendaban libros... No lo

hicieron... (Hombre, Derecho).

Conviene señalar, desde el punto de vista del examen sociolingüístico, dos aspectos diferentes. Por una parte, desde el punto de vista del relato de los entrevistados, nótese la pobreza de discurso en la expresión oral que puede validarse en el verbatim. En el primero de estos, por ejemplo, en una idea expresada en 74 palabras, esta persona utiliza ocho veces el vocablo "pues", a veces como conjunción, a veces a manera de interjección, etc. Lo mismo puede decirse de su empleo de la palabra "como". Es evidente, en las respuestas en general, el mal uso de la lengua, no solamente en lo que concierne a la lectoescritura, sino también en el sentido oral, lo cual podrá comprobarlo el lector en los testimonios citados en adelante. Por otra parte, desde el punto de vista del análisis del contexto social, nótese que en estas respuestas hay algunas constantes con relación a posibles causas de los niveles de literacidad y la formación y estimulación de los padres en el proceso de lectura y escritura: la madre o niñera como responsable de su proceso educativo y la no estimulación directa de los padres en el proceso de desarrollo de los niveles de literacidad.

Los entrevistados respondieron en la encuesta realizada al inicio de la investigación, que tenían buenos niveles de lectura y escritura, calificándose con cuatro puntos en una escala de uno a cinco, pero después de haber cursado el respectivo curso, su calificación varió considerablemente. De igual forma, hay una relación intrínseca entre los resultados obtenidos en las pruebas de entrada y de salida de los entrevistados, la formación de sus padres y los estratos socioeconómicos a los cuales pertenecen ${ }^{11}$.

En lo concerniente a la escuela primaria y su influencia en la "socialización secundaria", esta sería, desde una perspectiva idealista, el lugar donde los niños podrían subsanar las carencias o dificultades que tuvieron en el escenario familiar, en el proceso de construcción

11 Esta relación fue objeto de estudio de la presente investigación. Sin embargo, por cuestiones de espacio no es detallada en el presente artículo. La evidencia se describe y explica en LondoñoVásquez (2013). de buenos niveles de literacidad (Castañeda, \& Henao, 2002; Halliday, 1982). Esta creencia llevó al investigador principal a preguntarles cómo fue la relación con la lectura y la escritura en la escuela. Aquí algunos de sus testimonios: Pues en la escuela pues, como era, no enfatizaban tanto. Fue un colegio religioso pues o sea, no era tanto en lectura sino más que todo como religioso ¿cierto? Entonces de ahí vienen pues, venían muchas falencias (Hombre, Psicología). Pues, en la familia... pues mal ni mis papás nos enseñaron y en la escuela sí pero no mucha profundidad, era aprender el nombre desde primaria, leer las cartillitas pues de segundo y pero no fue como tan intensiva la lectura, entonces (Mujer, Derecho).

De cierta manera sí. Veíamos mucho documento y ya más que todo como eso... ¿Libros?, uno que otro, pues que de pronto me acuerde, me acuerdo del Principito, del Caballero de la armadura oxidada (Hombre, Derecho).

Las respuestas anteriores completan los datos de la investigación obtenidos en las pruebas escritas. Conviene insistir: en estas no solo es posible comprobar la dificultad que todavía tienen estos jóvenes para expresarse de manera oral, sino que, desde el punto de vista del análisis sociolingüístico, se podría afirmar que algunos de ellos todavía no han consolidado su "código elaborado". Recuérdese que esta noción, junto con la de "código restringido", son dos conceptos propuestos por Basil Bernstein (1989) con relación a las capacidades lingüísticas. Según este autor, estas capacidades son fuertemente dependientes de las diferentes prácticas sociales de las familias donde los niños se crían. En otras palabras, elementos como la formación de los padres o personas a cargo, la inclusión del niño en las discusiones, la opinión sobre temas de orden público, el acceso a fuentes de lectura (periódico, revistas y libros) y la argumentación en las respuestas dadas al niño son algunos elementos socioculturales que afectan directamente el desarrollo de sus capacidades lingüísticas. Un niño que carece de tales posibilidades tiende a desarrollar un código restringido; mientras 
que uno que accede a ellas, no solo puede ser funcional en el código restringido, sino también que lo hace en un código elaborado, código que es utilizado en los procesos de formación académica. En otras palabras, un estudiante que no maneje adecuadamente el código restringido, posiblemente tendrá problemas con su desempeño académico (Londoño-Vásquez \& Castañeda, 2011).

En el caso que nos asiste en la presente investigación, el análisis de las entrevistas permitió evidenciar la carencia del mencionado código elaborado, teniendo en cuenta que son jóvenes que han pasado por 11 años de formación académica, y el sistema de formación actual colombiano parece no brindarles las herramientas necesarias para desarrollar las competencias de literacidad requeridas para la educación superior.

Es importante anotar que el énfasis en los procesos de enseñanza/aprendizaje de la escuela primaria está puesto en contenidos de cartillas desarticuladas con las realidades de los estudiantes, en las cuales la ortografía y la puntuación siguen siendo los conceptos trabajados, más en la teoría que en la práctica, y cuyos resultados, que se pueden evidenciar en las pruebas de entrada y salida, siguen siendo negativos. Los procesos de lectura se llevan de forma desarticulada y no se motiva al estudiante para que realice este tipo de prácticas sociales en casa, con el fin de generar espacios de interacción y desarrollo.

De igual forma, parece que la secundaria no fue diferente para los dos hombres según pudo inferirse de sus respuestas:

Sí, y en la secundaria igual era pues, como proyectos de investigación entonces, ahí sí requería mucho pues, como mucho leer, investigar y todo el cuento, entonces ahí pues había como mucha, tenía muchas dificultades porque me tenía que esforzar demasiado. (Hombre, Psicología).

No. Yo estuve en este curso de Técnicas Americanas de Estudio, también estuve, pues inclusive lo terminé, hace ¿qué? ¿siete años?, mi mamá sí, mi mamá fue la que me incentivó por ese lado, ella lo hizo, allá más que todo se enfocan pues en la lectura rápida y en la comprensión...
Por ese lado si de pronto, cogí un poquito el hábito de leer, aunque no en la manera como lo enseñaron allá porque pa' eso hay que seguir práctica, hay que seguir uno practicando pues pa' no perder el hábito. Ya el resto pues creo que leo normal, si de pronto no como mucha gente que lee y repite en la mente, porque vos ves que es uno leyendo y no repitiendo... internamente (Hombre, Derecho).

Sin embargo, una de las estudiantes reconoce la importancia de la secundaria gracias al papel de uno de sus docentes de castellano, aunque afirma no haber aprovechado la oportunidad que le brindó dicho docente:

Sí, porque tenía un profesor muy bueno.

Tenía un profesor muy bueno de castellano y el tipo si nos mandaba a leer terrible. Pero, no leíamos los textos o copiábamos, entonces, no nos exigía tanto, solamente era como la nota (Mujer, Derecho).

De igual forma, los entrevistados reconocen haber tenido dificultades con los otros cursos de la malla curricular de su respectivo programa universitario como resultado de sus bajos niveles de literacidad.

\subsection{Entrevista a una participante extranjera}

Es necesario señalar que, al iniciar la intervención en el grupo del programa de Psicología, se consideró que esta participante no podría hacer parte de la unidad de análisis, puesto que sus niveles de literacidad eran muy bajos (su lengua materna no era el español). Además, llevaba pocos meses en Colombia y los primeros ejercicios en el curso la angustiaban tanto que le causaban lágrimas. No obstante, a las pocas semanas de estudio, los resultados que iba mostrando eran sorprendentes: aprendió a elaborar un resumen con base en las macrorreglas, identificaba los diferentes tipos de significados, mejoró su redacción, escribía textos coherentes y cohesionados, realizaba lecturas críticas, identificaba falacias argumentativas y ella misma argumentaba satisfactoriamente. Sus avances fueron tan valiosos que fue la participante con uno de los mejores resultados en la prueba de salida. 
Por consiguiente, al ser ella una de las voluntarias y al tener presente estos avances, se decidió entrevistarla, con el ánimo de conocer su contexto sociocultural, su valoración y las condiciones que le habían permitido desarrollar los niveles de literacidad en español.

Una de las respuestas más significativas se dio cuando se le preguntó si consideraba que ella tenía un buen nivel de lectura y escritura:

En español, no. En español, cuando yo llegué, pues a presentar las pruebas de admisión en la IUE me sentí como que perdida, llevaba como cinco meses acá y entonces no considero que tenía un buen nivel, pero hoy sí, ya me defiendo (Mujer Psicología).

Ciertamente, sus niveles de literacidad en español eran bajos, lo cual pudo además evidenciarse con el puntaje de 1,0 obtenido en la prueba de entrada. ¿Cómo alcanzó un puntaje final por encima 4,0? Es posible que los niveles de literacidad en su lengua materna (portugués) sean óptimos, y las dificultades iniciales estén relacionadas con el desconocimiento del vocabulario $\mathrm{y}$, en general, del funcionamiento del sistema lexicogramatical del español.

Pero si la dificultad en la prueba de entrada era solo el vocabulario, es posible que las variables socioculturales fueran positivas para el desarrollo de niveles de literacidad inicialmente en su lengua materna $\mathrm{y}$, posteriormente, en español. Por esto se le preguntó cómo era la relación en casa con respecto a la lectura y la escritura, a lo cual respondió:

Eh, yo creo que el contacto que tuve con la lectura y la escritura fue en el colegio, porque mi papá no fue alfabetizado, mi mamá solo hasta la primaria y mis hermanos tampoco se interesaron mucho por este camino académico, entonces yo soy la única que, que me fui por este camino, y en la escuela yo creo que las bases de todo son en la educación primaria. Yo tuve muy buenos docentes, que me incentivaron mucho (Mujer, Psicología).

$\mathrm{Su}$ testimonio permite inferir interesantes aspectos en dos niveles diferentes de su análisis. Por una parte, es claro que esta participante viene de una familia donde sus padres y hermanos no alcanzaron mayores logros académicos, algunos de ellos ni siquiera aprendieron a leer, pues, según ella, no les interesó. Entonces ¿es esta participante una mujer con capacidades extraordinarias o hubo otras variables socioculturales que le permitieron subsanar las dificultades en la familia? Es aquí donde emerge otro nivel de análisis sociolingüístico de la entrevista. Ella misma, al preguntarle por su relación en casa, responde que fue en el colegio, y no en el seno familiar, localizándose en otro contexto. Esto lo corrobora ella misma de inmediato, ya que a la pregunta ¿Cómo fue la relación con la lectura y la escritura en la escuela?, ella señaló lo siguiente:

Bueno, eh, desde la primaria, el contacto con la lectura fue eh - cómo te digofue como exigente. Nosotros teníamos todos los días media hora, eh, de lectura, y esa lectura se llevaba a la casa como tarea y se hacía a un ensayo para toda la próxima clase. Eh, entonces se volvió una tarea fácil. Pero cuando ingresas al bachillerato es diferente, o sea, son más materias son más son más contenidos y son, pues, todas más exigentes, no se resumen en simples ensayos, sino que eh, es una capacidad de lectura más exigente. No sé cómo te explicar... Yo creo que como a todos los niños, como nos enseñan los sonidos de los de las vocales, luego del alfabeto, de, de, las combinaciones que se hacen y, y, y normal, pues... en mi casa nunca fue incentivado mucho por ese lado por..., por la situación de mis papás, pero eso venía..., eso es un..., eso es intrínseco, ¿cierto?, que tú adquieres y yo hacía por mí misma, o sea, buscaba el conocimiento siempre, siempre. Siempre buscaba mucho conocimiento... Yo creo que mi docente de primaria, que se llamaba [nombre incomprensible] fue la primera docente que reconocía mis habilidades en la lectura y escritura, entonces me incentivó muchísimo, me prestaba sus libros, que ella tenía en su casa, una biblioteca muy grande, y me prestaban y además de los libros de, de la cual que teníamos en la biblioteca, 
entonces yo era como lo que llaman acá el ratón de biblioteca (Mujer, Psicología).

El examen de su respuesta permite señalar que, un proceso centrado en el acercamiento inicial a la lectura y escritura constante y dinámica, puede permitir que los niños vayan desarrollando poco a poco hábitos donde se requieren altos niveles de literacidad, y que construyan códigos elaborados para interactuar con los diferentes participantes de su realidad. Esto puede, como en el caso de esta estudiante, traducirse en elementos de desempeño académico, venciendo las dificultades generadas en su contexto primario, es decir, por la escasa o nula estimulación por parte de la familia.

Estos niveles de literacidad benefician directamente el desempeño de la participante en los diferentes cursos, puesto que ella asegura no tener muchos problemas en sus cursos de psicología, gracias al trabajo realizado con el docente encargado del curso "Taller de Lectura y Escritura I":

Por increíble que parezca, no tuve muchos problemas. Eh..., tuve ahí un docente espectacular en Taller de Lectura y Escritura, y me apoyó muchísimo. Cuando ingresé yo pensaba en desistir porque yo pensé que no sería capaz de pasar por eso, porque el nombre como que asustaba un poquito. ¿Taller, para mí? Pues para los otros (tal vez no), pero a mí sí me asustaba, pero el docente, la metodología que él utilizó fue excelente; o sea, traía textos y nosotros éramos quien después de la lectura que él traía de los textos construíamos artículos de ensayos, entonces la práctica, la práctica (Mujer, Psicología).

Nótese que esta respuesta a la entrevista arroja pistas que permiten inferir que la participante considera que estuvo favorecida en el contexto de aprendizaje particular que es objeto de la investigación. Por otro lado, es importante recalcar que para el docente colaborador su estudiante más sobresaliente fue ella:

Los estudiantes llegan con formaciones desiguales, con experiencias socioculturales distintas y con niveles de compromiso diversos. De ahí que al terminar el curso algunos alcancen resultados más altos que otros; además, había problemas con el trabajo, con la familia. Es importante resaltar que la persona que más avanzó fue una extranjera, cuyo compromiso fue excelente. Sin el compromiso del estudiante, estos cursos no alcanzan los objetivos propuestos (Docente

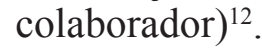

Al parecer, la metodología planteada por la intervención le facilitó a esta participante poder desarrollar y afianzar competencias lectoescriturales relacionadas con las diferentes áreas de la psicología, permitiéndole obtener un desempeño académico satisfactorio:

Eh, yo resumo en la metodología que empleó el docente. El grupo, fue dividido el grupo en dos, ¿cierto? Con dos docentes.

Yo tuve la oportunidad y el privilegio de estar con el docente [colaborador], y en cuanto a la metodología siempre nos traía textos de, eh, de los periódicos, y eran lecturas de bastante comprensión $y$ entonces de lectura crítica sobre todo. Entonces, ¿qué nos hacía? A través de esa metodología de aprender haciendo nosotros hacíamos muchos ensayos, [el profesor] se sentaba con cada uno y nos revisaba, nos mostraba los errores y luego ya dependería de cada uno eh, querer cambiarlos o no (Mujer, Psicología).

Finalmente, es interesante notar que esta participante pasó de obtener 1,0 en la prueba de entrada a 4,0 en la prueba de salida, entre otras cosas, gracias a una metodología pensada y diseñada para estudiantes cuya lengua materna es el español. Aunque hay que agregar que unas de las variables más importantes en este caso fue la de querer aprender, comprometerse consigo misma y participar en las actividades propuestas. De igual forma, las carencias lingüísticas originadas en la familia fueron subsanadas por docentes comprometidos que encontraron en la enseñanza de la lectura y la

12 En el proceso del curso, había cierta permisibilidad para entregar los informes y, sin embargo, algunos de los estudiantes nunca los entregaron. Por ejemplo, una nota del $10 \%$ era una autoevaluación y cinco jóvenes pertenecientes a la muestra, no la entregaron, a pesar de que la podían enviar por Internet. 
escritura la oportunidad de ayudar a la estudiante a consolidar conceptos y desarrollar habilidades y competencias en su lengua materna, las cuales después beneficiarían los procesos de literacidad en español como lengua extranjera. El análisis de esta entrevista permite ejemplificar la relación entre las variables sociales, culturales y lingüísticas, figurando el papel del contexto familiar y escolar, y su relación de cooperación, constitución y contribución en el desarrollo de las competencias lingüísticas.

\section{Conclusiones}

Las entrevistas cualitativas y el análisis sociolingüístico permitieron profundizar en la información recolectada para conocer los niveles de literacidad de los jóvenes universitarios que participaron en la investigación. Esto porque la entrevista cualitativa es una técnica que facilita al investigador acercarse a los participantes y ensayar a comprender, desde sus enunciados, como construyen su mundo, lo referencian y lo experimentan. Esta información es vital en el proceso descriptivo y explicativo. Por tanto, se convierte en una opción para complementar los datos sociolingüísticos de base.

Se puede afirmar entonces que el análisis sociolingüístico de las entrevistas semidirigidas en la investigación cualitativa resulta pertinente para examinar problemas como el de los niveles de literacidad de los estudiantes que comienzan sus estudios universitarios, en pro de diseñar intervenciones académicas específicas tendientes a subsanar, con mejores resultados, las falencias con las que llegan los participantes a iniciar su proceso de formación.

Esta es una de las implicaciones prácticas de la presente investigación. Aunque esta no obedeció a una intención meramente prescriptiva, el examen de la literatura, favoreció el diseño y aplicación de una intervención académica que revisó los enfoques tradicionales y adoptó un enfoque más del texto, a partir del Análisis Crítico del Discurso, la Argumentación Pragmadialéctica y la Lingüística Textual. Asimismo, gracias a la información recolectada a través de las entrevistas cualitativas y las encuestas socioculturales, se han podido fortalecer las prácticas pedagógicas de los investigadores involucrados y del docente colaborador, al igual que la coherencia de las temáticas y el desarrollo de estas en los cursos relacionados con la lectura y la escritura para ámbitos académicos.

Por otra parte, se pudo notar que el contexto biográfico desde la infancia y las instituciones involucradas como la familia y la escuela son preponderantes en la adquisición de las competencias en comprensión lectora, producción escritural y apropiación discursiva.

Una de las evidencias encontradas es que algunos de los jóvenes de la unidad de análisis no han desarrollado un código elaborado que les permita interactuar en el proceso de aprendizaje de cada uno de sus programas académicos. La prevalencia de un código restringido después de 11 años de educación, entre primaria y secundaria, puede ser el resultado de una propuesta educativa como la colombiana, donde hay promoción automática y mínimos de repitencia. En un país en que los estratos socioeconómicos que predominan son bajos y la formación académica de los padres es, en general, la de estudios secundarios, esto es un caldo de cultivo para la reproducción este tipo de código, como lo demostraron Bourdieu y Passeron (1996) hace ya más de tres décadas. Lo anterior permite señalar que el lenguaje en el proceso de socialización no solo tiende a favorecer algunos modos de aprendizaje por encima de otros, sino que crea también para ciertos jóvenes, entre el hogar y la escuela, una continuidad de cultura que en gran parte es negada a otros. Aquí reside la importancia de un análisis sociolingüístico de los niveles de literacidad de estos jóvenes.

Sin embargo, las limitaciones del estudio saltan a la vista. El presente, es un ejercicio preliminar en el sentido que presenta, únicamente, unos modestos hallazgos de una investigación con una muestra harto restringida en una única institución universitaria. A todas luces, como autores estamos en deuda aún en cuanto a la construcción de nueva teoría referente a los niveles de literacidad en la población universitaria y del apoyo metodológico del análisis sociolingüístico.

Futuras investigaciones podrían orientarse a continuar estudiando la influencia del contexto biográfico desde la infancia y la adquisición de 
este tipo de competencias indispensable para la juventud universitaria. Esto podría contribuir a refinar los procesos de enseñanza/aprendizaje de esta población.

\section{Lista de referencias}

Aguilar, J., Ramírez, A., \& López, R. (2014). Literacidad digital académica de los estudiantes universitarios: un estudio de caso. Revista Electrónica de Investigación y Docencia, 11, 123-146. Recuperado de: http://www.uv.mx/personal/albramirez/ files/2014/02/literacidad_reid.pdf

Bardin, L. (1977). L'analyse de contenu. Paris: Presses Universitaires de France.

Beaugrande, R. de, \& Dressler, W. U. (2007). Introducción a la lingüística de texto. Barcelona: Ariel.

Behrman, E. (2006). Teaching about language, power, and text: A review of classroom practices that support critical literacy. Journal of Adolescent and Adult Literacy, 49(6), 490-498. doi:10.1598/JAAL.49.6.4

Berger, P., \& Luckmann, T. (2003). La construcción social de la realidad. Buenos Aires: Amorrortu.

Bernstein, B. (1989). Clases, códigos y control: estudios teóricos para una sociología del lenguaje. Madrid: Akal.

Bourdieu, P., \& Passeron, J.-C. (1996). La reproducción: elementos para una teoría del sistema de enseñanza. México, D. F.: Fontamara.

Botzakis, S. (2016). Visual and digital texts. Journal of Adolescent \& Adult Literacy, 59(6), 731-733. doi:10.1002/ jaal.518

Boutet, J. (2016). Langues et enquêtes quantitatives: Introduction. Langage et Société, 155(1), 7-14. doi:10.3917/ 1s. 155.0007

Boyer, H. (2001). Introduction à la sociolinguistique. Paris: Dunod.

Buitrago, S., Ramírez, J., \& Ríos, J. (2011). Interferencia lingüística en el aprendizaje simultáneo de varias lenguas extranjeras. Revista Latinoamericana de Ciencias Sociales, Niñez y Juventud, 9(2), 721-737. Recuperado de: http://www.redalyc.org/ articulo.oa?id=77321592016
Calsamiglia, H., \& Tusón, A. (1999). Las cosas del decir. Barcelona: Ariel.

Cassany, D. (2005a). Los significados de la comprensión crítica. Lectura y Vida, 26(3), 32-45. Recuperado de:

https://repositori.upf.edu/bitstream/ handle/10230/22468/Cassany_lyv. pdf? sequence $=1$

Cassany, D. (2005b). Navegar con timón crítico. Cuadernos de Pedagogía, (352), 36-39. Recuperado de:

http://www.perio.unlp.edu.ar/catedras/ system/files/cassany_d._navegar_con_ timon_critico.pdf

Cassany, D. (2006). Tras las líneas. Sobre lectura contemporánea. Barcelona: Anagrama.

Cassany, D. (2008). Prácticas letradas contemporáneas. México, D. F.: Ríos de Tinta.

Castañeda, L. S., \& Henao, J. I. (2002). El papel del lenguaje en la apropiación de conocimiento. Bogotá, D. C.: Icfes.

Castek, J., \& Beach, R. (2013). Using apps to support disciplinary literacy and science learning. Journal of Adolescent \& Adult Literacy, 56(7), 554-564. Recuperado de: http://www.jstor.org/stable/41827900

Cook-Gumperz, J. (Comp.) (1988). La construcción social de la alfabetización. Barcelona: Paidós Ibérica.

Cyrulnik, B. (2001). Les vilains petits canards. Paris: Odile Jacob.

Forlot, G., \& Martin, F. (eds.) (2014). Regards sociolinguistiques contemporains: Terrains, espaces et complexités de la recherche. Paris: L'Harmattan.

Freebody, P., \& Luke, A. (1990). 'Literacies' programs: Debates and demands in cultural context. Prospect, 5(3), 7-16. Recuperado de: http://eprints.qut.edu.au/49099/1/ DOC090312.pdf

Friedman,A.M.(2016). Perceptual construction: Rereading. The social construction of reality through the sociology of the senses. Cultural Sociology, 10(1), 77-92. doi:10.1177/1749975515615149.

Green, P. (2001). Critical literacy revisited. En H. Fehring, \& P. Green (Eds.), Critical 
literacy: A collection of articles from the Australian Literacy Educators' Association (pp. 7-63). Newwark: Internacional Reading Association.

Halliday, M. A. K. (1982). El lenguaje como semiótica social. México, D. F.: Fondo de Cultura Económica.

Henao, J., Londoño-Vásquez, D. A., Frías, L., \& Castañeda, L. (2011). Niveles de literacidad de los estudiantes del programa de Psicología de la Institución Universitaria de Envigado. Zona Próxima, (15), 54-77. Recuperado de: file://D:/OneDrive $\% 20$ -\%20Fundaci\%C3\%B3n\%20CINDE/user/ Downloads/2176-12331-1-PB.pdf

Hernández-Rosete, D., \& Maya, O. (2016). Discriminación lingüística y contracultura escolar indígena en la Ciudad de México. Revista Latinoamericana de Ciencias Sociales, Niñez y Juventud, 14(2), 1161-1176. doi:10.11600/169271 5x.14219060815.

Holstein, J. A., \& Gubrium, J. F. (2016). Narrative practice and the active interview. En D. Silverman (Ed.), Qualitative Research (pp. 67-82). London: Sage.

Isáziga-David, C. H., Gabalán-Coello, J., \& Vásquez-Rizo, F. E. (2014). La intervención académica en la construcción de una sociedad con calidad: análisis del valor agregado en el proceso formativo colombiano. Hallazgos, 11(22), 359-384.

Jablonka, F. (2016). Gilles Forlot et Fanny Martin, regards sociolinguistiques contemporains: Terrains, espaces et complexités de la recherche (Carnets d'Atelier de Sociolinguistique, numéro hors série). Compte rendu. Zeitschrift für Romanische Philologie, 132(1), 287-296.

Jaramillo, A., Montaña, G., \& Rojas, L. (2006). Detección de errores en el proceso metacognitivo de monitoreo de la compresión lectora en niños. Revista Latinoamericana de Ciencias Sociales, Niñez y Juventud, 4(2), 75-95. Recuperado de: $\quad$ http://www.redalyc.org/articulo. oa? id=77340204

Kress, G. (2003). Literacy in the new media age. Nueva York: Routledge.
Kvale, S. (2011). Las entrevistas en investigación cualitativa. Madrid: Morata.

Labov, W. (1976). Sociolinguistique. Paris: Minuit.

Larson, L. C. (2013). It's time to turn the digital page: Preservice teachers explore e-book reading. Journal of Adolescent \& Adult Literacy, 56(4), 280-290. Recuperado de: http://www.jstor.org/stable/23367698

Lawson, R., \& Sayers, D. (eds.) (2016). Sociolinguistic research: Application and impact. London and New York: Routledge.

Londoño-Vásquez, D. A. (2013). Análisis sociolingüístico de los niveles de literacidad en jóvenes de la Institución Universitaria de Envigado. (Tesis doctoral no publicada). Universidad de ManizalesCinde, Manizales, Colombia.

Londoño-Vásquez, D. A. (2015). Jóvenes y literacidad: un análisis sociolingüístico. Envigado: Editorial Institución Universitaria de Envigado.

Londoño-Vásquez, D. A., \& Castañeda, L. S. (2011). Basil Bernstein y la relación lenguaje-educación: el caso del Semestre de Afianzamiento (SEA) de la Institución Universitaria de Envigado. Revista Lasallista de Investigación, 8(2), 18-32. Recuperado de: http://www.redalyc.org/ pdf/695/69522607003.pdf

Londoño-Vásquez, D. A., \& Bermúdez, H. L. (2013). Tres enfoques sobre los estudios críticos del discurso en el examen de la dominación. Palabra Clave 16(2), 491519. Recuperado de:

http://palabraclave.unisabana.edu. co/index.php/palabraclave/article/ view/3066/3193 doi:10.5294/ pacla.2013.16.2.9

Parker, J. K. (2013). Critical literacy and the ethical responsibilities of student media production. Journal of Adolescent \& Adult Literacy, 56(8), 668-676. Recuperado de: http://www.jstor.org/stable/41827921

Parodi, G. (Ed.) (2010). Alfabetización académica y profesional en el siglo XXI: leer y escribir desde las disciplinas. Santiago de Chile: Ariel.

Philliber, W. W., Spillman, R. E., \& King, R. (1996). Consequences of family literacy 
for adults and children: Some preliminary findings. Journal of Adolescent \& Adult Literacy, 39(7), 558-565. Recuperado de: http://www.jstor.org/stable/40017464

Renkema, J. (1999). Introducción a los estudios sobre el discurso. Barcelona: Gedisa.

Rowsell, J., \& Kendrick, M. (2013). Boys' hidden literacies: The critical need for the visual. Journal of Adolescent \& Adult Literacy, 56(7), 587-599. Recuperado de: http://www.jstor.org/stable/41827903

Sánchez-Castaño, J., Castaño-Mejía, O., \& Tamayo-Alzate, Ó. (2015). La argumentación metacognitiva en el aula de ciencias. Revista Latinoamericana de Ciencias Sociales, Niñez y Juventud, 13(2), 1153-1168. doi:10.11600/169271 5x.13242110214

Stahl, N. A., King, J. R., \& Eilers, U. (1996). Postsecondary reading strategies rediscovered. Journal of Adolescent \& AdultLiteracy, 39(5), 368-379. Recuperado de: http://www.jstor.org/stable/40014658

Van Dijk, T. (1991). La ciencia del texto. Barcelona: Paidós

Van Dijk, T. (1999). Ideología. Barcelona: Gedisa.

Van Eemeren, F., \& Grootendost, R. (1990). Analyzing argumentative discourse. En R. Trapp, \& J. Schuetz (Eds.), Perspectives on argumentation: Essays in honor of Wayne Brockriede (pp. 86-106). Prospect Heights: Waveland Press.

Van Eemeren, F., \& Grootendost, R. (1992). Argumentation, communication, and fallacies: A pragma-dialectical perspective. Hillsdale: Lawrence Erlbaum Associates.

Van Eemeren, F., Grootendost, R., \& Snoeck, F. (2006). Argumentación, comunicación $y$ falacias: una perspectiva pragmadialéctica. Santiago de Chile: Universidad Católica de Chile.

Vargas, A. (2015). Literacidad crítica y literacidades digitales: ¿una relación necesaria? (Una aproximación a un marco teórico para la lectura crítica). Revisar Folios, 42, 139-160.

Zavala, V. (2009) ¿Quién está diciendo eso?: literacidad académica, identidad y poder en la educación superior. En J. Kalman, \& B. Street (Eds.), Lectura, escritura y matemáticas como prácticas sociales: diálogos con América Latina (pp. 348363). México, D. F.: Siglo XXI. 